\title{
Treating Post-traumatic Stress Disorder with Neuromodulation Therapies: Transcranial Magnetic Stimulation, Transcranial Direct Current Stimulation, and Deep Brain Stimulation
}

\author{
Flavia Venetucci Gouveia ${ }^{1}$ - Benjamin Davidson ${ }^{2,3}$ - Ying Meng ${ }^{2,3}$ - Darryl Christopher Gidyk ${ }^{1}$ - Jennifer S. Rabin ${ }^{1,2,4}$. \\ Enoch $\mathrm{Ng}^{2,5} \cdot$ Agessandro Abrahao $^{1,2,4} \cdot$ Nir Lipsman $^{1,2,3} \cdot$ Peter Giacobbe ${ }^{1,2,5} \cdot$ Clement Hamani $^{1,2,3}$ (I)
}

Published online: 28 May 2020

(C) The American Society for Experimental NeuroTherapeutics, Inc. 2020

\begin{abstract}
Post-traumatic stress disorder (PTSD) is a prevalent and debilitating illness. While standard treatment with pharmacotherapy and psychotherapy may be effective, approximately 20 to $30 \%$ of patients remain symptomatic. These individuals experience depression, anxiety, and elevated rates of suicide. For treatment-resistant patients, there is a growing interest in the use of neuromodulation therapies, including transcranial magnetic stimulation (TMS), transcranial direct current stimulation (tDCS), and deep brain stimulation (DBS). We conducted a systematic review on the use of neuromodulation strategies for PTSD and pooled 13 randomized clinical trials (RCTs), 11 case series, and 6 case reports for analysis. Overall, most studies reported favorable outcomes in alleviating both PTSD and depressive symptoms. Although several RCTs described significant differences when active and sham stimulations were compared, others found marginal or nonsignificant differences between groups. Also positive were studies comparing PTSD symptoms before and after treatment. The side effect profile with all 3 modalities was found to be low, with mostly mild adverse events being reported. Despite these encouraging data, several aspects remain unknown. Given that PTSD is a highly heterogeneous condition that can be accompanied by distinct psychiatric diagnoses, defining a unique treatment for this patient population can be quite challenging. There has also been considerable variation across trials regarding stimulation parameters, symptomatic response, and the role of adjunctive psychotherapy. Future studies are needed to address these issues.
\end{abstract}

Key Words Post-traumatic stress disorder (PTSD) $\cdot$ neuromodulation $\cdot$ transcranial magnetic stimulation $(\mathrm{TMS}) \cdot$ transcranial direct current stimulation (tDCS) $\cdot$ deep brain stimulation (DBS)

Flavia Venetucci Gouveia and Benjamin Davidson contributed equally to this work.

Clement Hamani

clement.hamani@sunnybrook.ca

1 Sunnybrook Research Institute, 2075 Bayview Av, S126, Toronto, ON M4N3M5, Canada

2 Harquail Centre for Neuromodulation, Sunnybrook Health Sciences Centre, Toronto, ON M4N 3M5, Canada

3 Division of Neurosurgery, Sunnybrook Health Sciences Centre, University of Toronto, Toronto, ON M4N 3M5, Canada

4 Division of Neurology, Department of Medicine, Sunnybrook Health Sciences Centre, University of Toronto, Toronto, ON M4N 3M5, Canada

5 Department of Psychiatry, Sunnybrook Health Sciences Centre, University of Toronto, Toronto, ON M4N 3M5, Canada

\section{Introduction}

Post-traumatic stress disorder (PTSD) is a chronic psychiatric illness that develops as a pathologic response after exposure to a major traumatic event [1]. According to the National Institutes of Health $(\mathrm{NIH})$, the prevalence of PTSD in the community is $6.8 \%$, reaching approximately $20 \%$ among military veterans [2]. Conventional PTSD treatment consists of pharmacotherapy and psychotherapy. Presently, the only FDA-approved medications for PTSD are 2 selective serotonin reuptake inhibitors (SSRIs): paroxetine and sertraline [3]. Multiple psychotherapy approaches have been studied, although exposure therapy predominates [4]. Over the years, there has been a growing interest in neuromodulation strategies for the treatment of PTSD [5, 6]. In particular, numerous studies have been published employing transcranial magnetic stimulation (TMS), transcranial direct current stimulation 
(tDCS), and deep brain stimulation (DBS) [7-10]. These techniques allow for countless permutations of stimulation parameters and the possibility of applying concurrent therapies, such as exposure therapy [8]. In contrast to TMS and tDCS, DBS is an invasive neuromodulation technique. To date, only 1 study has reported the clinical effects of DBS in PTSD [7]. Understanding previous published studies on neuromodulation for PTSD could provide important insights for future trials.

\section{Methods}

This systematic review followed PRISMA guidelines (Fig. 1). A literature search was conducted for original articles with no limit for publication date up to December 2019 using PubMed/MEDLINE. The articles were required to match the following search terms: "Post-traumatic Stress Disorder," "PTSD," "Transcranial Magnetic Stimulation," "TMS," "Transcranial Direct-Current Stimulation," "tDCS," "Deep Brain Stimulation," and "DBS." The abstracts pulled in this search were reviewed by two independent investigators (FVG, BD). After excluding duplicates, relevant articles were selected for full-text review and had to meet the following inclusion and exclusion criteria: (I) inclusion (clinical studies of patients with PTSD treated with DBS, TMS, or tDCS; articles published in English) and (II) exclusion (studies including only preclinical data; articles reporting on patient populations other than PTSD; review articles; commentaries; technical notes or protocols). When articles reported duplicate data, only the study reporting the most recent follow-up results was included.

\section{Results and Discussion}

As a prevalent and often treatment-resistant condition, PTSD is a major concern and has been the focus of numerous studies aiming to develop neuromodulation therapies (e.g., vagus nerve stimulation [11-13], stellate ganglion block [14, 15], electroconvulsive therapy $[16,17]$, TMS $[9,10]$, and tDCS $[8,18])$. Most of these treatments have focused on increasing prefrontal inhibitory control over the amygdala, either directly or indirectly, to reduce the excessive emotional response to an aversive memory related to the trauma $[6,19]$. Along a similar line, psychotherapies, such as exposure therapy, are based on protocols of fear extinction that gradually reduce the excessive emotional response by repeatedly presenting a trigger of the traumatic event in the absence of the threatening situation $[6$, 11]. The use of neuromodulation therapies in association with psychotherapy may lead to greater extinction recall and, consequently, an improvement of symptoms $[6,19,20]$.
PTSD is also frequently characterized by comorbid major depressive disorder [21]. It should be noted that many treatment modalities and targets have also been employed in the treatment of depression. Clinical benefit in PTSD patients could have derived in part from an improvement of depressive symptoms, although most studies were designed to treat PTSD directly rather than comorbidities.

With this rationale, several studies evaluated the feasibility and the clinical benefits of using TMS or tDCS alone or combined with psychotherapy. In this review, we evaluated 13 RCTs $[9,10,18,21-30], 11$ case series [8, 31-40], and 6 case reports [7, 21, 41-44] on neuromodulation for PTSD, focusing on TMS, tDCS, and DBS. Details of the articles pooled for analysis in our review are summarized in Tables 1 (TMS), 2 (tDCS), and 3 (DBS).

\section{Transcranial Magnetic Stimulation}

TMS is a safe, noninvasive neuromodulatory technique that uses electromagnetic fields to change local electrical activity and alter neuronal firing patterns resulting in widespread connectivity modifications [30]. Although the mechanisms of action of TMS are not fully understood, it is believed that the use of different stimulation frequencies (high frequency, $>10 \mathrm{~Hz}$; low frequency, $\leq 1 \mathrm{~Hz}$ ) results in an increase or decrease in cortical excitability [38]. Variations of the TMS protocol are theta burst stimulation (TBS) and synchronized TMS. TBS is intended to elicit long-term potentiation by using theta band rhythm stimulation, normally performed with high-frequency $(50 \mathrm{~Hz})$ TMS bursts with inter-burst intervals of $200 \mathrm{~ms}$ [45]. Synchronized TMS uses the individualized oscillatory neuronal activity in the alpha frequency band information to generate stimulation settings that are believed to restore the normal oscillatory neuronal and increase neural plasticity [40]. When used repeatedly, repetitive TMS (rTMS) becomes a powerful tool for treating psychiatric disorders with no need for anesthesia [38]. As a result, this technique has been proposed as a suitable therapeutic alternative for some neuropsychiatric disorders [46].

Several clinical trials have been published on the therapeutic effect of TMS for PTSD with promising results. In general, these studies report a significant reduction in PTSD symptoms when comparing active versus sham stimulation, or before versus after treatment conditions [10, 23, 28]. Studies investigating the therapeutic response of distinct TMS settings (e.g., low $v s$ high frequency) have shown similar results, with a larger effect in patients receiving low-frequency stimulation $[26,27]$. Also, it has been suggested that a larger therapeutic effect is observed in patients treated with right dorsolateral prefrontal cortex (dIPFC) stimulation compared with left dlPFC stimulation [24], though no difference was found when right versus bilateral dIPFC stimulation was compared [30]. 
Fig. 1 PRISMA flow chart describing record selection.

\section{PRISMA Flow Chart - Records Selection}

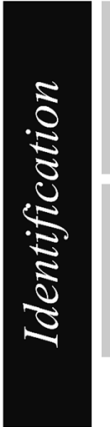

Search Terms: Post-traumatic Stress Disorder; PTSD; Transcranial Magnetic Stimulation; TMS; Transcranial Direct Current Stimulation; tDCS; Deep brain Stimulation; DBS.

Records identified through database search $(n=471)$

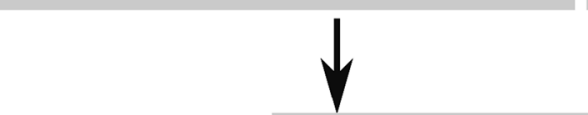

Records after duplicates were removed $(\mathrm{n}=158)$
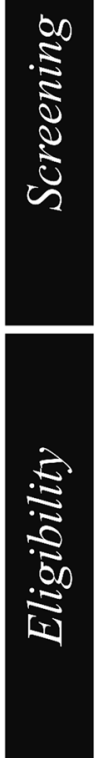

Records Screend $(n=158)$

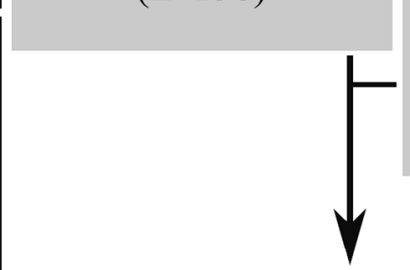

Full-text articles assessed for eligibility $(n=61)$
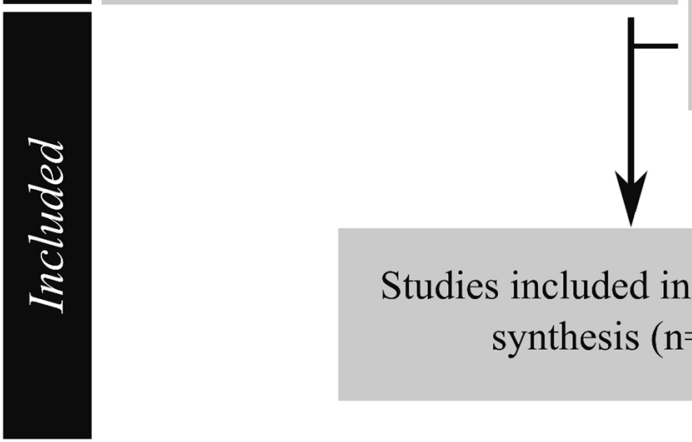

Review/Letters/Comments/Protocols $(n=72)$

Written in othel language $(n=9)$

Preclinical data $(\mathrm{n}=16)$

Total $(n=97)$

\section{Studies included in qualitative} synthesis $(n=30)$
Although the majority of the studies were performed using TMS alone, some clinical trials investigated the effects of stimulation along with psychotherapy (e.g., cognitive processing therapy; exposure therapies). In general, these studies reported significant improvements in patients receiving combined treatments $[9,21,22,25]$.

One important aspect of noninvasive neuromodulation therapies (e.g., TMS and tDCS) is the low side effect profile
[47]. Most studies on TMS reported transient mild side effects that were easily treated with standard pharmacotherapy (e.g., headache, insomnia, and mood disturbances) $[10,21,27,30]$. Nevertheless, 4 cases of severe adverse events were documented. These included suicidality, homicidal ideation, tonic-clonic generalized seizures, and a probably unrelated case of subconjunctival hemorrhage in a patient with a clinical history of ocular disease [22, 28, 31, 41]. 


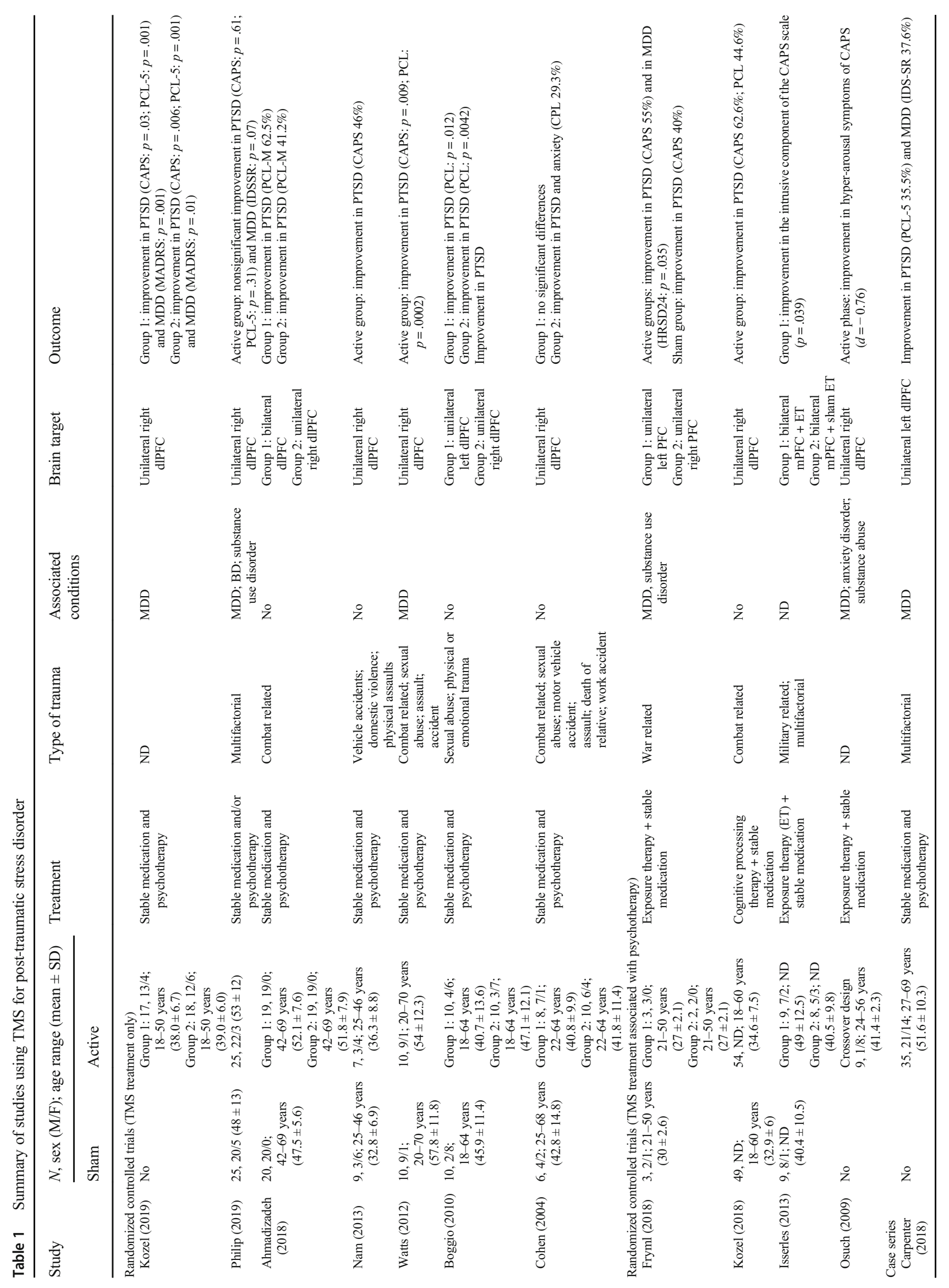




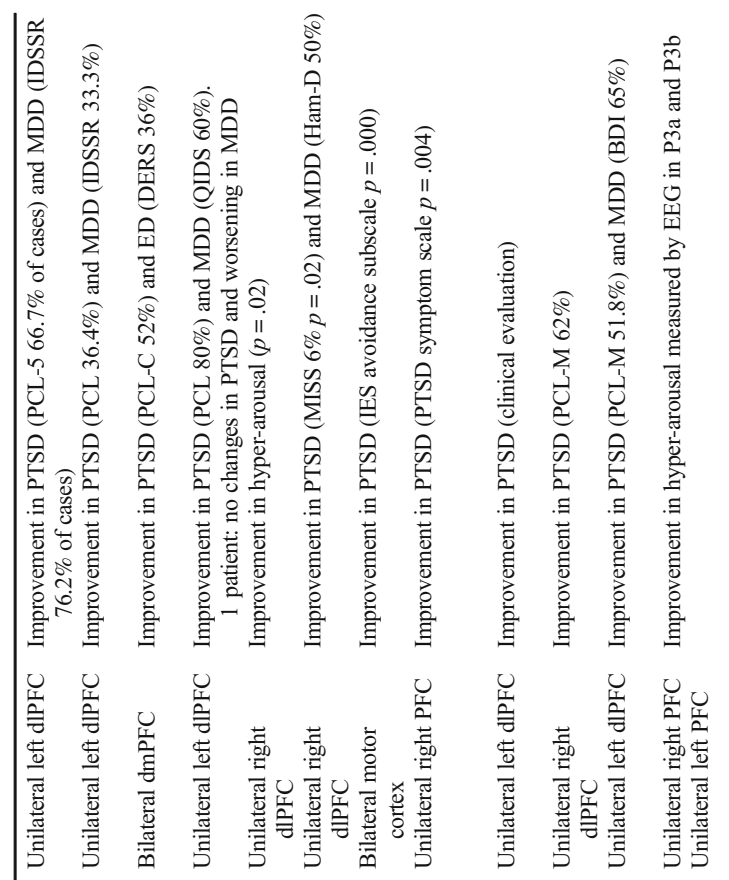

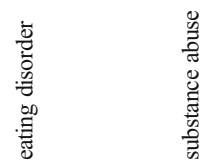

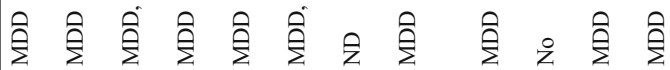

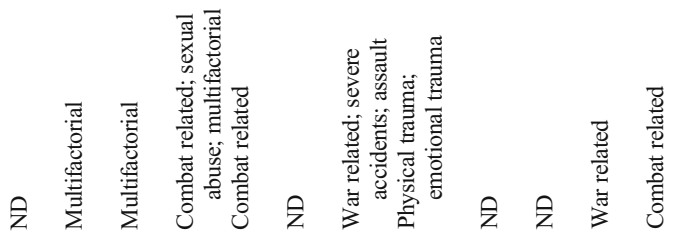

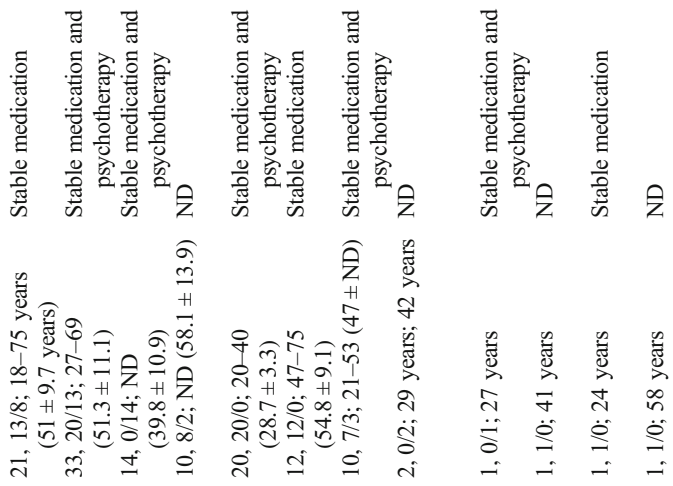

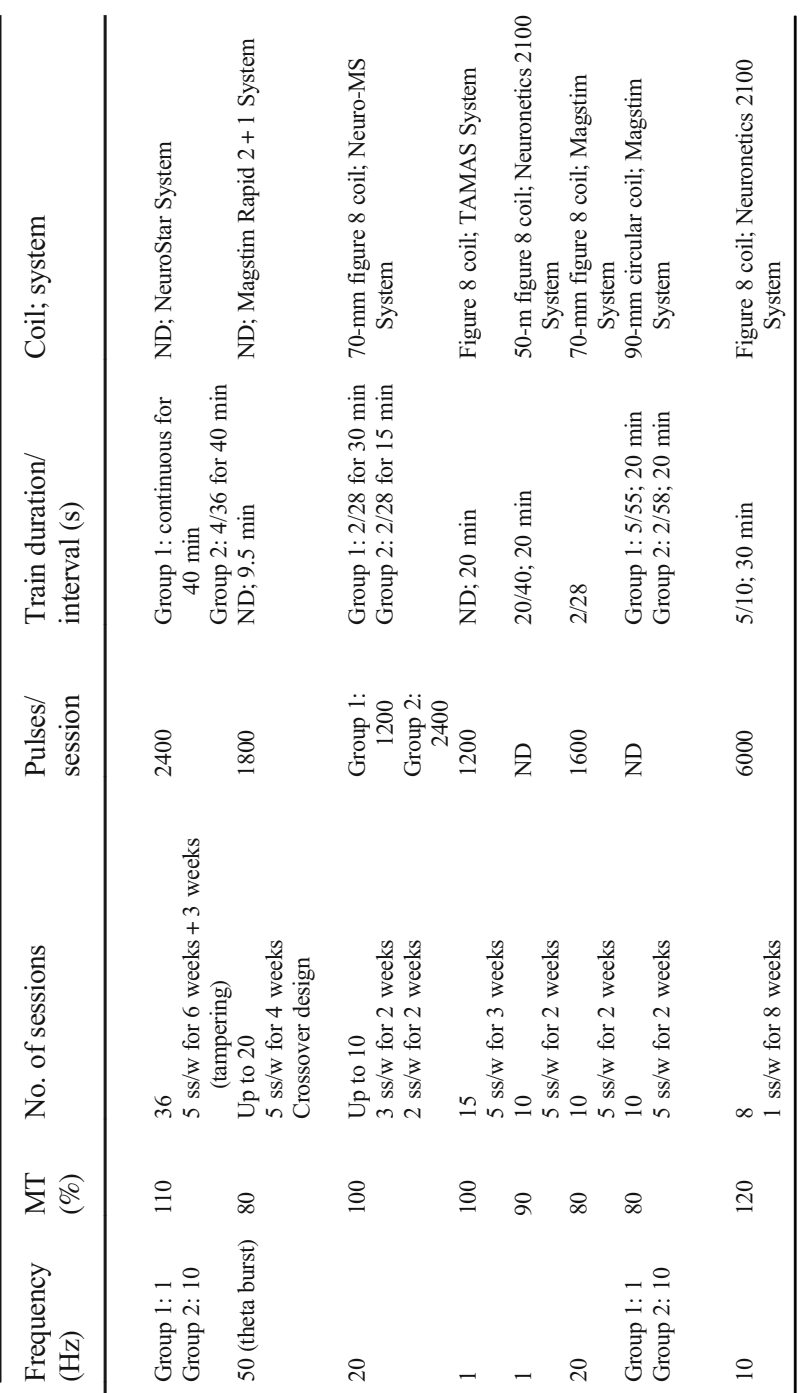

0
0
0
0
0
0
0
0

言

苞

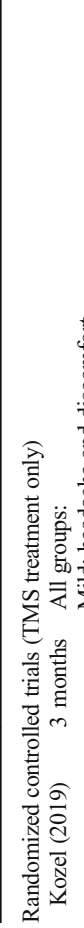

in

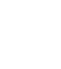

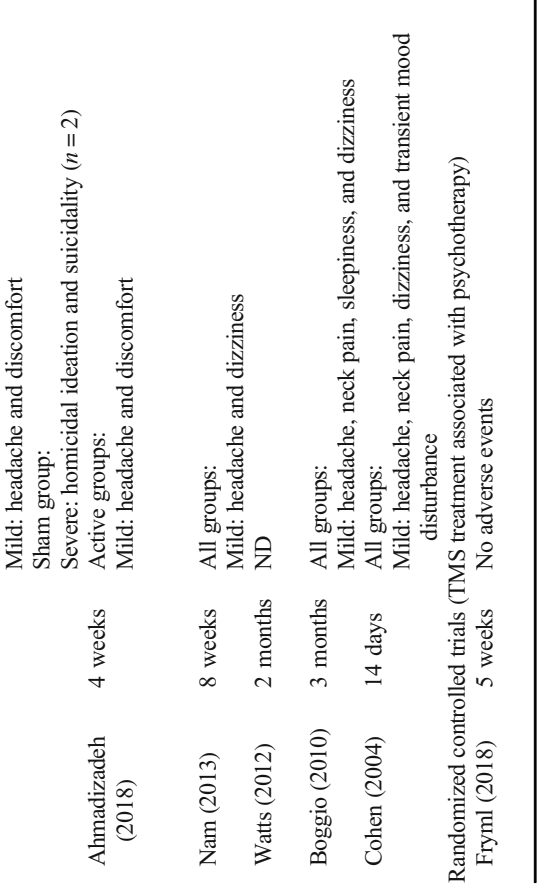




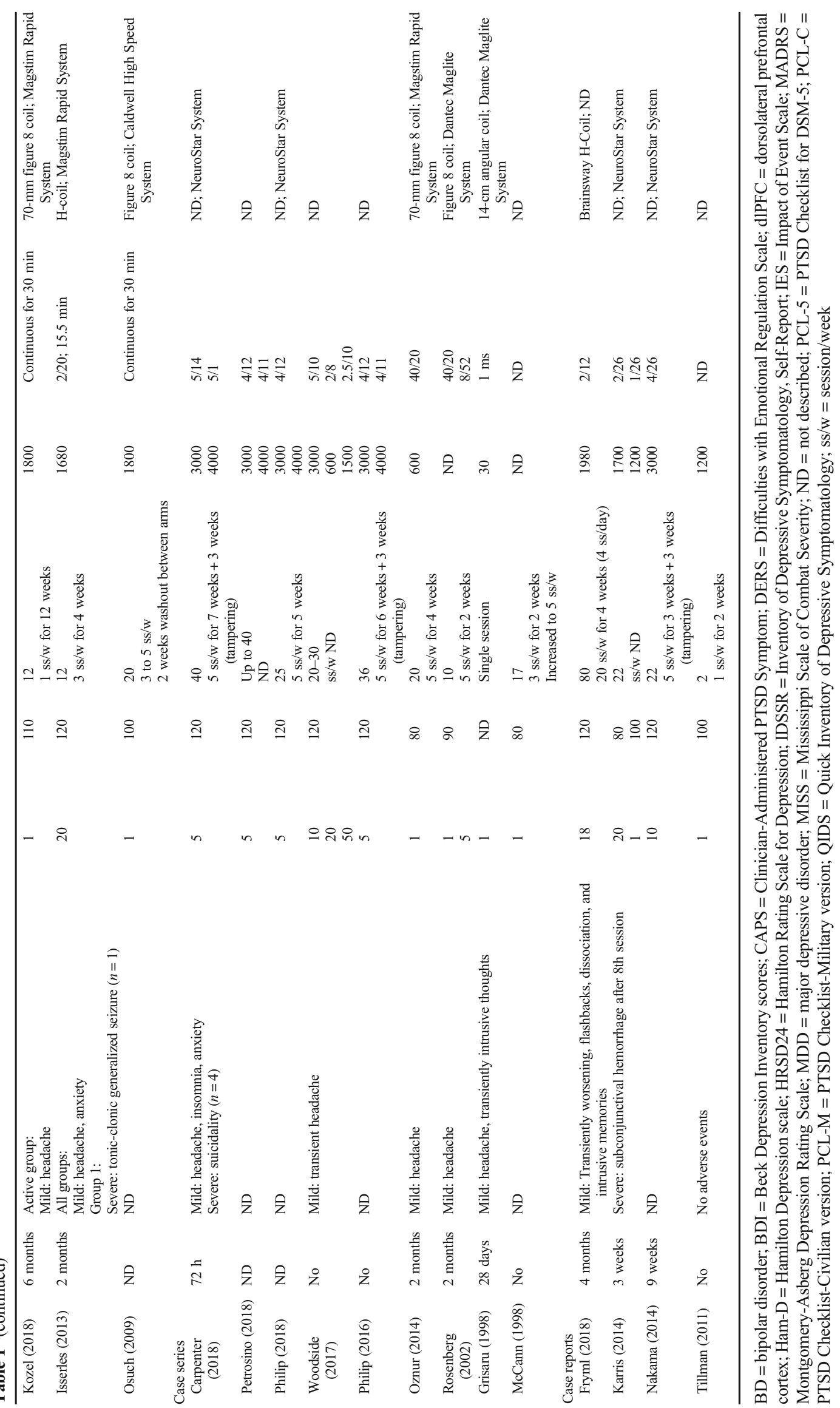


It is important to highlight that TMS studies included patients with multiple types of trauma (e.g., combat-related, vehicle accidents, sexual violence), with a wide age range (18-75 years) and a slight predominance of males (Table 1). Furthermore, several patients had comorbidities such as major depressive disorder (most frequent), substance abuse, and eating disorder [25, 33, 34]. The brain target (e.g., dlPFC, medial prefrontal cortex, dorsomedial prefrontal cortex) and the stimulation parameters varied greatly across studies. TMS settings ranged from 1 to $20 \mathrm{~Hz}$ with intensities of 80 to $120 \%$ of the motor threshold (Table 1), with the exception of a study that used intermittent TBS [40, 47].

\section{Transcranial Direct Current Stimulation}

Similar to TMS, tDCS is a safe and noninvasive neuromodulation therapy capable of altering local electrical activity, resulting in the restoration of normal connectivity between dysfunctional neurons [8]. While TMS uses focused electromagnetic pulses (trains of stimulation), tDCS uses constant and low direct current $[8,30]$ to increase or decrease cortical excitability by depolarizing (anodal) or hyperpolarizing (cathodal) neurons [48, 49]. There are fewer studies investigating the beneficial effects of tDCS for the treatment of PTSD than those evaluating TMS. Thus, the clinical evidence of the therapeutic effects of this technique relies on two clinical trials and 3 case series (Table 2).

A pilot study published in 2015 investigated the feasibility of treating PTSD symptoms with a combination of computerized working memory training and prefrontal cortex tDCS [39]. In this case series, the authors report significant improvements on cognitive and emotional performance measures that were accompanied by neurophysiological changes observed on electroencephalographic recordings [8]. Thereafter, another pilot study investigated the feasibility of using ventromedial prefrontal cortex (vmPFC) tDCS during extinction learning to improve extinction recall in PTSD patients [8]. Using normalized skin conductance reactivity as a primary outcome measure, the authors showed that vmPFC-tDCS is more effective during extinction consolidation than when applied simultaneously with extinction learning [8]. This suggests that this strategy may be an interesting way of enhancing extinction

Table 2 Summary of studies using tDCS for post-traumatic stress disorder

\begin{tabular}{|c|c|c|c|c|c|}
\hline Study & $\begin{array}{l}\text { Ahmadizadeh } \\
\text { (2019) }\end{array}$ & $\begin{array}{l}\text { van’t Wout-Frank } \\
\text { (2018) }\end{array}$ & Hampstead (2019) & van’t Wout (2017) & Saunders (2015) \\
\hline Study type & RCT & $\mathrm{RCT}$ & Case series & Case series & Case series \\
\hline $\begin{array}{l}\text { TDCS + } \\
\text { psychotherapy }\end{array}$ & No & Exposure therapy & No & Fear conditioning & Working memory training \\
\hline $\begin{array}{l}\text { Sham group, } N \text {, sex, } \\
\text { age range in years } \\
\text { (mean } \pm \mathrm{SD})\end{array}$ & $\begin{array}{l}20,6 \text { males } \\
\quad(43.00 \pm 2.42)\end{array}$ & $\begin{array}{l}6 \text {, all males, } 30-53 \\
\quad(40.5 \pm 8.8)\end{array}$ & No & No & No \\
\hline $\begin{array}{l}\text { Active group, } N \text {, sex, } \\
\text { age range in years } \\
(\text { mean } \pm \mathrm{SD})\end{array}$ & $\begin{array}{l}20,8 \text { males } \\
\quad(44.50 \pm 2.34)\end{array}$ & $\begin{array}{l}\text { 6, all males, } 30-53 \\
(40.5 \pm 8.8)\end{array}$ & 6, 4 males, 20-65 (ND) & $\begin{array}{l}\text { 28, all males, } 30-69 \\
\quad(56.25 \pm 12.3)\end{array}$ & 4, all males, 55-65 (ND) \\
\hline Treatment & $\begin{array}{l}\text { Stable medication } \\
\text { and psychotherapy }\end{array}$ & $\begin{array}{l}\text { Stable medication } \\
\text { and psychotherapy }\end{array}$ & Stable medication & Stable medication & ND \\
\hline Type of trauma & ND & War zone related & $\begin{array}{l}\text { Combat related; sexual trauma; } \\
\text { traumatic death }\end{array}$ & War zone related & ND \\
\hline Associated conditions & MDD & ND & MDD, GAD & Anxiety disorder & ND \\
\hline Brain target & dlPFC & vmPFC & rLTC & vmPFC & $\mathrm{PFC}$ \\
\hline Outcome & $\begin{array}{l}\text { Improvement in } \\
\text { PTSD (PCL-5: } \\
p=.00)\end{array}$ & $\begin{array}{l}\text { Improvement in } \\
\text { PTSD (PCL-5: } \\
p=.03 \text { ) }\end{array}$ & $\begin{array}{c}\text { Improvement in PTSD (CAPS } \\
\text { 7-57\%; PCL-C 19-38\%) }\end{array}$ & $\begin{array}{l}\text { Improvement in } \\
\text { PTSD (PCL-5: } \\
p=.02 \text { ) }\end{array}$ & $\begin{array}{l}\text { Improvement in cognition and } \\
\text { emotion (clinical evaluation) }\end{array}$ \\
\hline Follow-up & 30 days & 1 month & 3-4 days & $24 \mathrm{~h}$ & 10 weeks \\
\hline \multirow[t]{2}{*}{ Side effects } & All groups: & Active group: & All groups: & Active group: & ND \\
\hline & $\begin{array}{l}\text { Mild: Itching, } \\
\text { Tingling }\end{array}$ & Mild & Mild: itching, tingling, burning & Mild: erythema & \\
\hline \multicolumn{6}{|l|}{ Stimulation settings } \\
\hline Current (mA) & 2 & 20 & 2 & 2 & 10 \\
\hline No. of sessions & $\begin{array}{c}10(5 \mathrm{ss} / \mathrm{w} \text { for } \\
2 \text { weeks })\end{array}$ & $\begin{array}{c}6(3 \mathrm{ss} / \mathrm{w} \text { for } \\
2 \text { weeks })\end{array}$ & Up to $10(5 \mathrm{ss} / \mathrm{w}$ for 2 weeks $)$ & $2(\mathrm{ND})$ & 5 (ND) \\
\hline Duration (min) & 20 & 25 & 20 & 10 & 20 \\
\hline Anode/cathode & $\mathrm{F} 3 / \mathrm{F} 4$ & $\mathrm{AF} 3 / \mathrm{PO} 8$ & F8; C4; P8; EX10/T8 & $\mathrm{AF} 3 / \mathrm{PO} 8$ & $\mathrm{AF} 3 / \mathrm{M} 1$ \\
\hline System & $\begin{array}{l}\text { neuroConn DC } \\
\text { Stimulator Plus }\end{array}$ & $\begin{array}{l}\text { neuroConn DC } \\
\text { Stimulator Plus }\end{array}$ & ND & $\begin{array}{l}\text { neuroConn DC } \\
\text { Stimulator Plus }\end{array}$ & Soterix Medical 1224-B \\
\hline
\end{tabular}

CAPS = Clinician-Administered PTSD Scale for DSM-IV; dIPFC $=$ dorsolateral prefrontal cortex GAD = generalized anxiety disorder; MDD = major depressive disorder; ND = not described; PCL-C = PTSD Checklist-Civilian version; $\mathrm{PFC}=$ prefrontal cortex; $\mathrm{RCT}=$ randomized controlled trial; $\mathrm{rLTC}=$ right lateral temporal cortex; $\mathrm{ss} / \mathrm{w}=$ sessions/week; $\mathrm{vmPFC}=$ ventromedial prefrontal cortex 
Table 3 Summary of the study using DBS for post-traumatic stress disorder

\begin{tabular}{ll}
\hline Study & Langevin (2016) \\
Study type & Case report \\
Sham group & No \\
Active group & 1 male, 48 years \\
Treatment & ND \\
Type of trauma & War zone related \\
Associated conditions & No \\
Brain target & Bilateral basolateral amygdala \\
Outcome & Improvement in PTSD (CAPS 37.8\%) \\
Follow-up & 8 months \\
Side effects & Transient mild mood alteration \\
Stimulation settings & Amplitude right, 1.4 V; amplitude left, 0.7 V; pulse width, $60 \mu$ s; frequency, $160 \mathrm{~Hz}$ \\
\hline
\end{tabular}

recall and improving PTSD symptoms [8]. Based on these findings, a double-blind randomized control trial was performed to further investigate extinction recall in patients with PTSD using vmPFC-tDCS along with sessions of a combatrelated virtual reality paradigm. The study reported significant reductions in arousal and severity of PTSD in patients allocated to the active group receiving exposure therapy [29]. More recently, a RCT evaluating the efficacy of dIPFC-tDCS alone for PTSD symptoms was published reporting a significant reduction in PTSD, depressive, and anxiety symptoms in the active group immediately after treatment and after a 1-month follow-up period [18].

These results are encouraging, as the therapeutic effects of tDCS were generally positive and the observed side effects were mild (Table 2). Nevertheless, it is important to highlight that most of studies recruited only patients with combatrelated PTSD, with a vast majority of male individuals $(71 \%)$. Also, age ranged from 20 to 69 years and most patients presented comorbidities, such as major depressive disorder and generalized anxiety disorder (Table 2). Furthermore, the anatomical target and the stimulation parameters were distinct between studies, including the PFC, PFC subregions, and temporal cortex, with varying current intensities (e.g., 2 to $20 \mathrm{~mA}$ ) and session duration (e.g., 10 to $25 \mathrm{~min}$ ).

\section{Deep Brain Stimulation}

DBS is an invasive neuromodulation technique that consists of the placement of electrodes in deep brain targets, followed by the delivery of electrical stimulation to modulate local and neural activity [50]. DBS has been extensively used to treat movement disorders [51-53] and is currently delivered through a humanitarian exemption for the treatment of obsessive-compulsive disorder in the USA, while it has CE marking and is approved in Europe $[54,55]$. There is one case report using DBS for the treatment of PTSD (Table 3). A 48year-old man with treatment refractory combat-related PTSD received bilateral DBS to the basolateral amygdala. The authors reported over $35 \%$ symptomatic improvement after 8 months of stimulation with no major adverse events [7].

\section{Conclusion}

Although numerous studies highlight the possible beneficial use of rTMS and tDCS for the treatment of PTSD, the exact mechanism of action remains unclear. It seems that the most effective target for rTMS is the right dIPFC and that more than 5 sessions are necessary for significant improvement. Within the range of tested settings, stimulation intensity or frequency did not seem to be important contributors to clinical outcome. tDCS, on the other hand, can be used as a psychotherapy treatment enhancer and facilitate symptom reduction.

Various studies included in this review reported favorable outcomes in alleviating both PTSD and depressive symptoms. Although several RCTs described significant differences when active and sham stimulation were compared, others found marginal or nonsignificant differences between groups. The fact that patients receiving sham treatment were often found to be improved highlights the magnitude of the placebo effect in this population. Longer follow-up periods will be necessary to better characterize not only the placebo response but also the duration of the therapeutic benefit and possible signs of refractoriness/relapse after neuromodulation treatment [56-59].

A positive and encouraging note in the studies reviewed was the low side effect profile of neuromodulation strategies in PTSD. The vast majority of articles reported absent or mild adverse events. Of the 636 patients included in the 30 studies pooled for review, only four developed serious adverse event $[22,28,31,41]$. All of these occurred in TMS studies, likely due to the disproportionate number of TMS-treated patients $(n=424)$ compared to tDCS $(n=90)$ and DBS $(n=1)$. 
Given that PTSD is a highly heterogeneous condition that can be accompanied by distinct psychiatric diagnoses, defining a unique treatment for this patient population can be quite challenging. There has also been considerable variation across trials regarding stimulation parameters, symptomatic response, and the role of adjunctive psychotherapy. Future studies will need to adopt standardized targeting and stimulation parameters and focus on well-defined PTSD subgroups.

Acknowledgments This work was supported in part with funds from the DANA Foundation, Veterans Affairs Canada, and the Harquail Centre for Neuromodulation. The authors declare no conflict of interest related to this work. $\mathrm{CH}$ was part of an unrelated advisory board for Medtronic.

Required Author Forms Disclosure forms provided by the authors are available with the online version of this article.

\section{References}

1. American Psychiatric Association. Diagnostic and Statistical Manual of Mental Disorders (DSM-5®). American Psychiatric Pub; 2013.

2. Richardson LK, Frueh BC, Acierno R. Prevalence estimates of combat-related post-traumatic stress disorder: critical review. Aust N Z J Psychiatry 2010;44:4-19.

3. Akiki TJ, Abdallah CG. Are There Effective Psychopharmacologic Treatments for PTSD? [Internet]. J Clin Psychiatry. 2018. Available from: https://doi.org/10.4088/jcp.18ac12473.

4. Grasser LR, Javanbakht A. Treatments of Posttraumatic Stress Disorder in Civilian Populations [Internet]. Curr Psychiatry Rep. 2019. Available from: https://doi.org/10.1007/s11920-019-0994-3.

5. Reznikov R, Binko M, Nobrega JN, et al. Deep Brain Stimulation in Animal Models of Fear, Anxiety, and Posttraumatic Stress Disorder. Neuropsychopharmacology. 2016;41:2810-2817.

6. Gouveia FV, Gidyk DC, Giacobbe P, et al. Neuromodulation Strategies in Post-Traumatic Stress Disorder: From Preclinical Models to Clinical Applications. Brain Sci [Internet]. 2019;9. Available from: https://doi.org/10.3390/brainsci9020045.

7. Langevin J-P, Koek RJ, Schwartz HN, et al. Deep Brain Stimulation of the Basolateral Amygdala for TreatmentRefractory Posttraumatic Stress Disorder. Biol Psychiatry 2016;79:e82-e84.

8. Van't Wout M, Longo SM, Reddy MK, et al. Transcranial direct current stimulation may modulate extinction memory in posttraumatic stress disorder. Brain Behav 2017;7:e0681.

9. Kozel FA, Motes MA, Didehbani N, et al. Repetitive TMS to augment cognitive processing therapy in combat veterans of recent conflicts with PTSD: A randomized clinical trial. J Affect Disord 2018;229:506-514.

10. Nam D-H, Pae C-U, Chae J-H. Low-frequency, Repetitive Transcranial Magnetic Stimulation for the Treatment of Patients with Posttraumatic Stress Disorder: a Double-blind, Shamcontrolled Study. Clin Psychopharmacol Neurosci 2013;11:96102.

11. Noble LJ, Meruva VB, Hays SA, et al. Vagus nerve stimulation promotes generalization of conditioned fear extinction and reduces anxiety in rats. Brain Stimul 2019;12:9-18.

12. Peña DF, Childs JE, Willett $S$, et al. Vagus nerve stimulation enhances extinction of conditioned fear and modulates plasticity in the pathway from the ventromedial prefrontal cortex to the amygdala. Front Behav Neurosci 2014;8:327.
13. George MS, Ward HE Jr, Ninan PT, et al. A pilot study of vagus nerve stimulation (VNS) for treatment-resistant anxiety disorders. Brain Stimul 2008;1:112-121.

14. Rae Olmsted KL, Bartoszek M, Mulvaney S, et al. Effect of Stellate Ganglion Block Treatment on Posttraumatic Stress Disorder Symptoms: A Randomized Clinical Trial. JAMA Psychiatry 2019;1-9.

15. Hanling SR, Hickey A, Lesnik I, et al. Stellate Ganglion Block for the Treatment of Posttraumatic Stress Disorder: A Randomized, Double-Blind, Controlled TrialReg Anesth Pain Med 2016;41: 494-500.

16. Ahmadi N, Moss L, Hauser P, et al. Clinical outcome of maintenance electroconvulsive therapy in comorbid Posttraumatic Stress Disorder and major depressive disorder. J Psychiatr Res 2018;105: 132-136.

17. Kaster TS, Goldbloom DS, Daskalakis ZJ, et al. Electroconvulsive therapy for depression with comorbid borderline personality disorder or post-traumatic stress disorder: A matched retrospective cohort study. Brain Stimul 2018;11:204-212.

18. Ahmadizadeh MJ, Rezaei M, Fitzgerald PB. Transcranial direct current stimulation (tDCS) for post-traumatic stress disorder (PTSD): A randomized, double-blinded, controlled trial. Brain Res Bull 2019;153:273-278.

19. Koek RJ, Roach J, Athanasiou N, et al. Neuromodulatory treatments for post-traumatic stress disorder (PTSD). Prog NeuroPsychopharmacol Biol Psychiatry 2019;92:148-160.

20. VanElzakker MB, Staples-Bradley LK, Shin LM. The Neurocircuitry of Fear and PTSD [Internet]. Sleep and CombatRelated Post Traumatic Stress Disorder. 2018. p. 111-125. Available from: https://doi.org/10.1007/978-1-4939-7148-0_10.

21. Fryml LD, Pelic CG, Acierno R, et al. Exposure Therapy and Simultaneous Repetitive Transcranial Magnetic Stimulation: A Controlled Pilot Trial for the Treatment of Posttraumatic Stress Disorder. J. ECT [Internet]. 2018; Available from: https://doi.org/ 10.1097/YCT.0000000000000505.

22. Isserles M, Shalev AY, Roth Y, et al. Effectiveness of deep transcranial magnetic stimulation combined with a brief exposure procedure in post-traumatic stress disorder-a pilot study. Brain Stimul 2013;6:377-383.

23. Watts BV, Landon B, Groft A, et al. A sham controlled study of repetitive transcranial magnetic stimulation for posttraumatic stress disorder. Brain Stimul 2012;5:38-43.

24. Boggio PS, Rocha M, Oliveira MO, et al. Noninvasive brain stimulation with high-frequency and low-intensity repetitive transcranial magnetic stimulation treatment for posttraumatic stress disorder. J Clin Psychiatry 2010;71:992-999.

25. Osuch EA, Benson BE, Luckenbaugh DA, et al. Repetitive TMS combined with exposure therapy for PTSD: a preliminary study. J Anxiety Disord 2009;23:54-59.

26. Cohen H, Kaplan Z, Kotler M, et al. Repetitive transcranial magnetic stimulation of the right dorsolateral prefrontal cortex in posttraumatic stress disorder: a double-blind, placebo-controlled study. Am J Psychiatry 2004;161:515-524.

27. Kozel FA, Andrew Kozel F, Van Trees K, et al. One hertz versus ten hertz repetitive TMS treatment of PTSD: A randomized clinical trial [Internet]. Psychiatry Research. 2019. p. 153-162. Available from: https://doi.org/10.1016/j.psychres.2019.01.004.

28. Philip NS, Barredo J, Aiken E, et al. Theta-Burst Transcranial Magnetic Stimulation for Posttraumatic Stress Disorder. Am J Psychiatry 2019;176:939-948.

29. van't Wout-Frank M, Shea MT, Larson VC, et al. Combined transcranial direct current stimulation with virtual reality exposure for posttraumatic stress disorder: Feasibility and pilot results. Brain Stimul. [Internet]. 2018; Available from: https://doi.org/10.1016/j. brs.2018.09.011. 
30. Ahmadizadeh, Rezaei. Unilateral right and bilateral dorsolateral prefrontal cortex transcranial magnetic stimulation in treatment post-traumatic stress disorder: A randomized controlled study. Brain Res Bull 2018;140:334-340.

31. Carpenter LL, Conelea C, Tyrka AR, et al. 5 Hz Repetitive transcranial magnetic stimulation for posttraumatic stress disorder comorbid with major depressive disorder. J Affect Disord 2018;235: 414-420.

32. Philip NS, Barredo J, van't Wout-Frank M, et al. Network Mechanisms of Clinical Response to Transcranial Magnetic Stimulation in Posttraumatic Stress Disorder and Major Depressive Disorder. Biol Psychiatry. 2018;83:263-272.

33. Woodside DB, Colton P, Lam E, et al. Dorsomedial prefrontal cortex repetitive transcranial magnetic stimulation treatment of posttraumatic stress disorder in eating disorders: An open-label case series. Int J Eat Disord 2017;50:1231-1234.

34. Philip NS, Ridout SJ, Albright SE, et al. 5-Hz Transcranial Magnetic Stimulation for Comorbid Posttraumatic Stress Disorder and Major Depression. J Trauma Stress 2016;29:93-96.

35. Oznur T, Akarsu S, Celik C, et al. Is transcranial magnetic stimulation effective in treatment-resistant combat related posttraumatic stress disorder? Neurosciences. 2014;19:29-32.

36. Rosenberg PB, Mehndiratta RB, Mehndiratta YP, et al. Repetitive transcranial magnetic stimulation treatment of comorbid posttraumatic stress disorder and major depression. J Neuropsychiatr Clin Neurosci 2002;14:270-276.

37. Hampstead BM, Mascaro N, Schlaefflin S, et al. Variable symptomatic and neurophysiologic response to HD-tDCS in a case series with posttraumatic stress disorder. Int. J. Psychophysiol. [Internet]. 2019; Available from: https://doi.org/10.1016/j.ijpsycho.2019.10. 017.

38. Grisaru N, Amir M, Cohen $\mathrm{H}$, et al. Effect of transcranial magnetic stimulation in posttraumatic stress disorder: a preliminary study. Biol Psychiatry 1998;44:52-55.

39. Saunders N, Downham R, Turman B, et al. Working memory training with tDCS improves behavioral and neurophysiological symptoms in pilot group with post-traumatic stress disorder (PTSD) and with poor working memory. Neurocase. 2015;21:271-278.

40. Petrosino NJ, Zandvakili A, Carpenter LL, et al. Pilot Testing of Peak Alpha Frequency Stability During Repetitive Transcranial Magnetic Stimulation. Front Psychiatry 2018;9:605.

41. Karris BC, Capobianco M. Subconjunctival hemorrhage after high frequency right-sided repetitive transcranial magnetic stimulation. Brain Stimul 2014;7:494-495.

42. Nakama H, Garcia A, O'Brien K, et al. Case report of a 24-year-old man with resolution of treatment-resistant major depressive disorder and comorbid PTSD using rTMS. J ECT 2014;30:e9-e10.

43. Tillman GD, Kimbrell TA, Calley CS, et al. Repetitive Transcranial Magnetic Stimulation and Threat Memory: Selective Reduction of Combat Threat Memory P300 Response After Right Frontal-Lobe Stimulation. J Neuropsychiatr 2011;23:40-47.

44. McCann UD. Repetitive Transcranial Magnetic Stimulation for Posttraumatic Stress Disorder [Internet]. Archives of General Psychiatry. 1998. p. 276-279. Available from: https://doi.org/10. 1001/archpsyc.55.3.276.
45. Oberman L, Edwards D, Eldaief M, et al. Safety of theta burst transcranial magnetic stimulation: a systematic review of the literature. J Clin Neurophysiol 2011;28:67-74.

46. Gonsalvez I, Baror R, Fried P, et al. Therapeutic Noninvasive Brain Stimulation in Alzheimer's Disease. Curr Alzheimer Res 2017;14: 362-376.

47. Rossi S, Hallett M, Rossini PM, et al. Safety, ethical considerations, and application guidelines for the use of transcranial magnetic stimulation in clinical practice and research. Clin Neurophysiol 2009;120:2008-2039.

48. Reato D, Salvador R, Bikson M, et al. Principles of Transcranial Direct Current Stimulation (tDCS): Introduction to the Biophysics of tDCS [Internet]. Practical Guide to Transcranial Direct Current Stimulation. 2019. p. 45-80. Available from: https://doi.org/10. 1007/978-3-319-95948-1 2.

49. Thair H, Holloway AL, Newport R, et al. Transcranial Direct Current Stimulation (tDCS): A Beginner's Guide for Design and Implementation [Internet]. Frontiers in Neuroscience. 2017. Available from: https://doi.org/10.3389/fnins.2017.00641.

50. Dandekar MP, Fenoy AJ, Carvalho AF, et al. Deep brain stimulation for treatment-resistant depression: an integrative review of preclinical and clinical findings and translational implications. Mol Psychiatry 2018;23:1094.

51. Guzzi G, Della Torre A, Chirchiglia D, et al. Critical reappraisal of DBS targeting for movement disorders. J Neurosurg Sci 2016;60: 181-188.

52. Krishnan S, Pisharady KK, Divya KP, et al. Deep brain stimulation for movement disorders. Neurol India 2018;66:S90-S101.

53. Lozano AM, Hutchison WD, Kalia SK. What Have We Learned About Movement Disorders from Functional Neurosurgery? Annu Rev Neurosci 2017;40:453-477.

54. Rapinesi C, Kotzalidis GD, Ferracuti S, et al. Brain Stimulation in Obsessive-Compulsive Disorder (OCD): A Systematic Review. Curr Neuropharmacol 2019;17:787-807.

55. Graat I, Figee M, Denys D. The application of deep brain stimulation in the treatment of psychiatric disorders. Int Rev Psychiatry 2017;29:178-190.

56. Mallinckrodt $\mathrm{CH}$, Zhang L, Prucka WR, et al. Signal detection and placebo response in schizophrenia: parallels with depression. Psychopharmacol Bull 2010;43:53-72.

57. Agid O, Siu CO, Potkin SG, et al. Meta-regression analysis of placebo response in antipsychotic trials, 1970-2010. Am J Psychiatry 2013;170:1335-1344.

58. Rutherford BR, Pott E, Tandler JM, et al. Placebo response in antipsychotic clinical trials: a meta-analysis. JAMA Psychiatry 2014;71:1409-1421.

59. Mancini M, Wade AG, Perugi G, et al. Impact of patient selection and study characteristics on signal detection in placebo-controlled trials with antidepressants [Internet]. J Psychiatr Res. 2014. p. 2129. Available from: https://doi.org/10.1016/j.jpsychires.2014.01. 001.

Publisher's Note Springer Nature remains neutral with regard to jurisdictional claims in published maps and institutional affiliations. 